\title{
Lecciones tras el desastre de Lorca (terremotos del 11 de mayo de 2011)
}

El 11 de mayo de 2011 tembló la tierra con fuerza en el sureste de la península Ibérica. Dos movimientos sísmicos de elevada magnitud ocurridos en la tarde de esa tarde, con su epicentro en las proximidades de la ciudad y a escasa profundidad de la superficie terrestre, provocaron diez víctimas mortales y elevadas pérdidas económicas debido a los destrozos causados en viviendas, mobiliario urbano y destrucción de patrimonio historico-artístico de tanta importancia en la capital del campo de Lorca.

Cuando ocurre una catástrofe natural lo peor, sin duda, son las víctimas causadas; las elevadas pérdidas económicas siempre son recuperables, aunque en esta ocasión pasarán años hasta que la ciudad de Lorca pueda recuperar su estado previo a los seísmos de esa jornada de mayo. Las estimaciones de pérdidas económicas realizadas en los días posteriores a la secuencia de terremotos elevan los daños a 700 millones de euros, de los cuales 50 millones corresponderían a las pérdidas ocasionadas en el rico patrimonio histórico-artístico de la ciudad.

Ante la magnitud del desastre, algunas lecciones pueden - y deben- sacarse, a modo de guía de trabajo que permita actuar en el futuro ante fenómenos de esta naturaleza en un país de riesgo como el nuestro y siempre con el objetivo de la reducción de la vulnerabilidad y la exposición a los episodios naturales de rango extremo.

1.- El terremoto sigue siendo el peligro natural de más difícil predicción entre los episodios de rango extraordinario. Ocurre en segundos y no deja se- 
ñales previas. En Lorca se produjo un primer movimiento de 4,5 grados de magnitud (equivalente a VI en la escala de intensidad), a las 17:05 h. (hora civil) del día 11 de mayo de 2011 que inició una serie de movimientos telúricos más o menos intensos que perdurarían hasta la jornada del 22 de mayo. Es decir, se trata de un período de actividad prolongado - 11 días- lo que indica que el juego de presiones y acomodos de los bloques geológicos afectados en este caso fue muy activo. Entre el primer movimiento en la tarde del 11 de mayo y el último significativo en la tarde del 22 de mayo se produjeron 124 sacudidas. La tierra, por tanto, no dejó de temblar durante casi dos semanas.

El terremoto más intenso ocurrió a las 18:47 h. (hora civil) del 11 de mayo; apenas casi dos horas depués del primer movimiento indicado. En esta ocasión la magnitud alcanzó 5,1 (equivalente a un grado VII en la escala de intensidad). Tanto el primero como el segundo pueden considerarse terremotos importantes y destructivos. Esa misma jornada, por la noche —a las 22:37 h. (hora civil) — tiene lugar una tercera sacudida sísmica importante de magnitud 3,9 (equivalenta a un grado IV enla escala de intensidad). Un dato importante en este episodio extremo es que los epicentros de estos tres movimientos sísmicos mayores se localizan en las proximidades de la propia ciudad (a 3,5 $\mathrm{km}, 2,9 \mathrm{~km}$ y $4,9 \mathrm{~km}$, respectivamente) y con una profundidad escasa respecto al nivel de superficie, de ahí que la transferencia de energía liberada por las sacudidas afectasen de inmediato al núcleo urbano y ocasionaran desplazamientos de terreno importantes.

Debe recordarse que Lorca está emplazada en una zona sísmica activa; en el área geológica de las Béticas, que forma una extensa franja de contacto entre las placas africana y europea (subsector Ibérico). Es una zona de enfrentamiento geológico continuo desde la era terciaria cuando, con los movimientos orogénicos alpinos, se configuró la coordillera Bética con sus distintas unidades. En concreto, Lorca está situada en la denominada Fosa Intrabética (falla Cádiz-Alicante) que marca la separación entre las unidades Subbéticas interiores y las Béticas stricto sensu que ocupan el territorio más próximo al litoral en el sureste peninsular. Además, el sector central de la fosa intrabética, entre Granada y Murcia, es el área más sismicamente activa que ha registrado, históricamente, los movimientos telúricos más activos (vid. figura 1).

2.- La historia nos proporciona también su lección. Cuando aconteció este terremoto del 11 de mayo de 2011, las noticias que transmitieron los medios de comunicación en los primeros momentos traducían una falta absoluta de conocimientos geográficos. Parecía que el terremoto de Lorca era algo nuevo, 
FIGURA 1

LOCALIZACIÓN DE TERREMOTOS IMPORTANTES OCURRIDOS EN LORCA Y LOS TERRITORIOS PRÓXIMOS EN EL SURESTE IBÉRICO

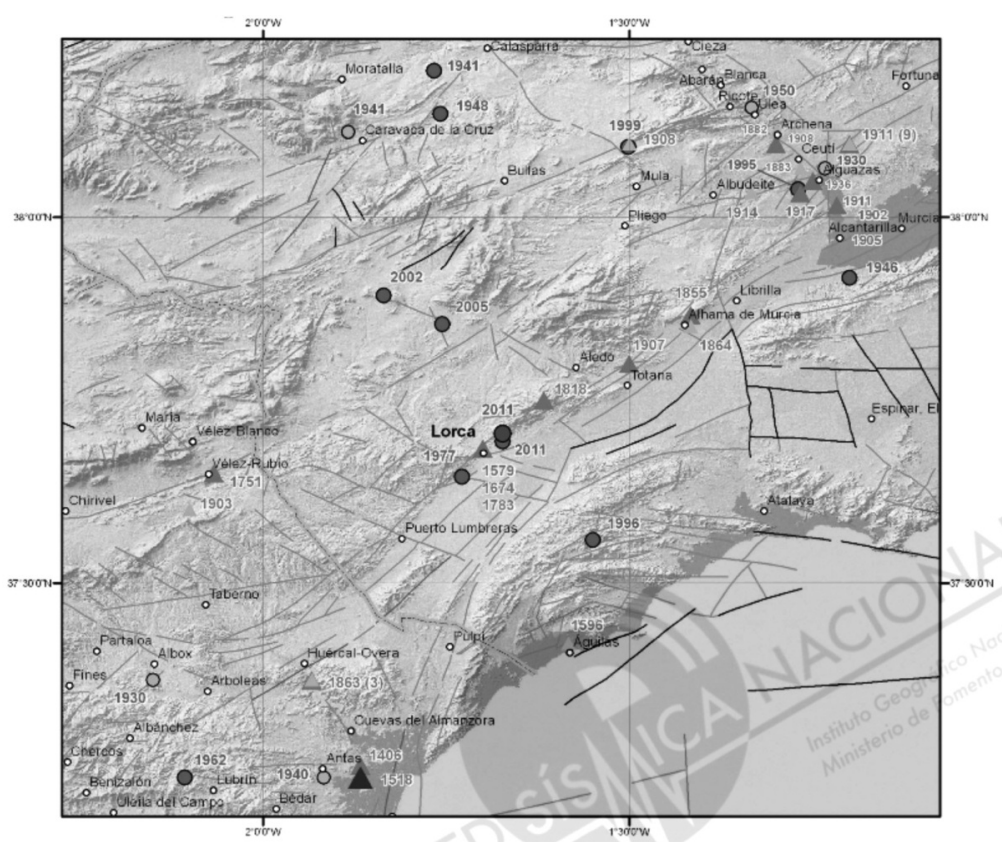

Fuente: Red Sísmica Nacional. Instituto Geográfico Nacional.

un peligro geológico apenas conocido y registrado en la zona y, en ningún caso, se tenía recuerdo de movimientos sísmicos ocurridos en el área geográfica del Lorca y sus alrededores que hubiesen alcanzado una magnitud similar a la anotada en este caso. Como se ha señalado, Lorca está ubicada en una franja geológica de constante actividad sísmica en el sureste peninsular, con múltiples fallas de contacto y de deslizamiento en su entorno, de manera que no debe sorprender el desarrollo de movimientos más o menos intensos en este espacio geográfico. En realidad, la tierra no deja de moverse nunca en el sureste Ibérico y se registran movimientos sísmicos a diario aunque de magnitud imperceptible por el ser humano. En el área próxima a Lorca, el registro histórico de la Red Sísmica Nacional del Instituto Geográfico Nacional tiene datados medio centenar de terremotos importantes ocurridos desde el siglo $\mathrm{xv}$, entre los que destacan los sucedidos en la propia ciudad de Lorca y que se recogen en la tabla 1. 
TABLA 1

TERREMOTOS IMPORTANTES OCURRIDOS EN LORCA

\begin{tabular}{ll}
\hline Fecha & INTENSIDAD (magnitud) \\
\hline $30 / 01 / 1579$ & VII \\
$10 / 08 / 1674$ & V-VI \\
$28 / 08 / 1674$ & VIII \\
$29 / 03 / 1783$ & VI-VII \\
$20 / 12 / 1818$ & VI-VII \\
$06 / 06 / 1977$ & VI $(4,2)$ \\
\hline
\end{tabular}

Fuente: Red Sísmica Nacional. Instituto Geográfico Nacional.

3.- La vida en territorios de riesgo debe conllevar la asunción de dicho riesgo por parte de la población asentada ${ }^{1}$. Bien sea por peligros de causa atmosférica o, como en este caso, geológica, los espacios de riesgo requieren un tratamiento especial en la planificación territorial. El sureste peninsular es el territorio con mayor riesgo natural de España (vid. figura 2). En ello confluyen unos valores elevados de los diferentes componentes del riesgo; esto es, una peligrosidad natural alta (sismicidad, inundaciones y sequías, como peligros más importantes que allí se manifiestan) y una vulnerabilidad y exposición al riesgo también elevada (territorio con elevada población y ocupación intensa del suelo). En el caso de Lorca - y es una característica aplicable al conjunto del litoral mediterráneo español- el riesgo, antes de haberse reducido en las últimas décadas, se ha incrementado de consuno a una mayor ocupación territorial motivada por el crecimiento urbano de la propia ciudad, por la urbanización del campo de Lorca y por el importante incremento de población registrado en los últimos veinte años. En el municipio de Lorca la población de hecho ha pasado de 66.437 hab. en 1988 a 92.692 hab. en 2010; de esta cifra, 60.000 personas viven en la propia ciudad de Lorca, el resto en las pedanías que componen su extenso término municipal. Se da la circunstancia añadida de que una proporción importante de la población que se ha instalado en la ciudad de Lorca es inmigrante. Se trata de colectivos poblacionales procedentes del norte de Africa o de Sudamérica (especialmente, de Ecuador) que han venido a este territorio de oportunidad a trabajar en la actividad agraria,

\footnotetext{
${ }^{1}$ Olcina Cantos, J. (2011): "Un mundo de riesgo". Lychnos, Cuadernos de la Fundación General CSIC, 4, marzo, Madrid, pp. 54-60.
} 
Figura 2

SÍNTESIS DE LOS PELIGROS NATURALES EN ESPAÑA

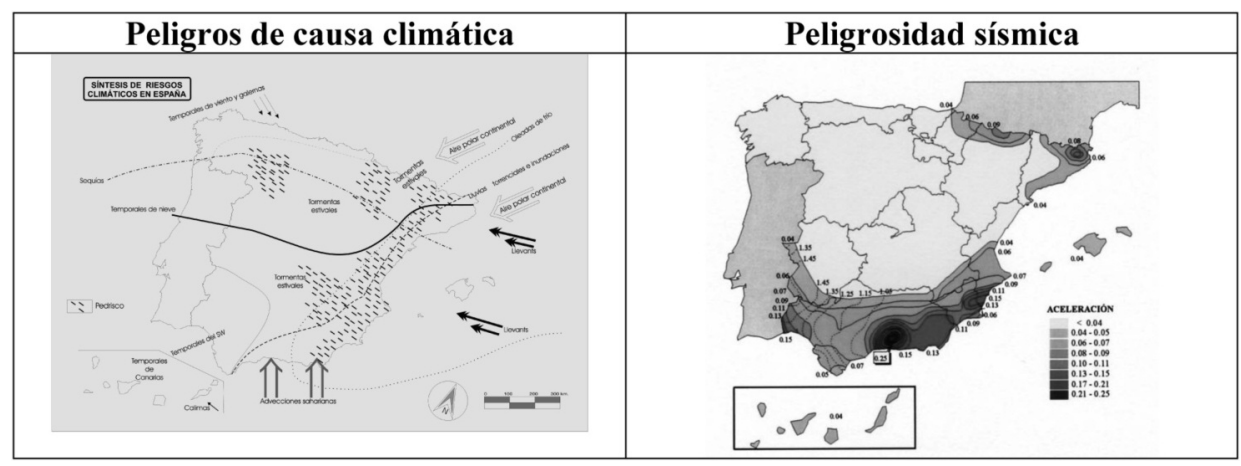

Fuente: IGN y elaboración propia.

en la industria o en los servicios de la ciudad. Según datos del padron municipal, en 2010, el 20\% de la población total del municipio de Lorca era inmigrante. Es la manifestación local de un proceso general que ha afectado a buena parte del litoral mediterráneo español desde la década de los años noventa del pasado siglo. Y esto añade un nuevo factor de riesgo: este contigente de población desconoce que Lorca es un territorio de riesgo, porque nadie se ha preocupado en educarlos para el riesgo. Como tampoco a la población autóctona. El fenómeno de la inmigración laboral y residencial de las dos últimas décadas en nuestro país y el aumento de la superficie artificial en espacios de peligrosidad natural innata ha supuesto un aumento del riesgo en nuestro país. De manera que no es exagerado afirmar que en España, y especialmente en el litoral mediterráneo y el archipiélago canario hay, en la actualidad, más riesgo frente a un episodio natural de rango extraordinario que hace diez o veinte años. Y ello a pesar de la aparición de normativa ambiental y territorial que incorpora el tratamiento del riesgo como medida de reducción de la exposición a los peligros naturales.

4.- España ha desarrollado investigación de calidad sobre sismicidad y ha implantado sistemas de detección de movimientos sísmicos que están a la altura de los países más avanzados en este tema del mundo occidental. Existe una buena red sísmica nacional que se completa con mediciones que se llevan a cabo en laboratorios de investigación universitaria. En el caso del terremoto del Lorca ha habido un seguimiento puntual y detallado por parte del Instituto Geográfico Nacional y se ha difundido la información obtenida en dicha 
red al gran público, aspecto que habla a favor de la profesionalidad de los investigadores y técnicos de la Red Sísmica $\mathrm{Nacional}^{2}$ y de la superación de recelos en la transparencia de la comunicación de datos que, en cuestiones de riesgo, siempre beneficia a la seguridad de la ciudadanía.

5.- A resultas de los destrozos ocurridos en los inmuebles de la ciudad de Lorca, puede señalarse que la norma de construcción sismorresistente que existe en España desde 1972, ha resultado insuficiente, amen protocolo de control del cumplimiento de dicha norma que deberá ser modificado. Recordemos que desde 1972 se han promulgado diverentes versiones de la norma sismorresistente hasta la actualmente vigente de 2002 (Real Decreto 997/2002, de 27 de septiembre, por el que se aprueba la norma de construcción sismorresistente: parte general y edificación, NCSR-02). Esta normativa establece la obligación de construir con determinadas precauciones antisísmicas en función del nivel de peligrosidad sísmica existente en un territorio. El problema es que asigna un único valor para dicha peligrosidad, a partir de la aceleración sísmica esperada, para todo un término municipal. Esto es un error porque la constitución geológica en un municipio es muy diversa y la respuesta a un movimiento sísmico de un edificio o una infraestructura no es la misma si el sustrato es calcareo, margoso o arenoso. De manera que son necesarios estudios de microzonación sísmica que permitan adecuar las exigencias constructivas a la respuesta real que puede tener un terremoto en el espacio geográfico. En el caso de Lorca, además, es necesario señalar que estamos ante uno de los términos municipales más extensos de España y con realidades geológicas muy diferenciadas, por tanto el valor único de aceleración sísmica que le otorga la normativa es inapropiado. La norma sismorresistente debe adecuarse a la nueva realidad, proporcionada por la investigación sísmica, de la elaboración de análisis y cartografía de gran precisión escalar (microzonación sísmica). Y también, debe adaptarse para adecuar las construcciones a presiones sísmicas muy intensas en caso de terremotos superficiales, como ha ocurrido en Lorca el 11 de mayo. Hay otro dato importante: en ciudades con rico patrimonio histórico-artístico, como lo es Lorca, hay que arbitrar medidas especiales para la protección sísmica de estos edificios. Como se ha señalado, los daños económicos en los edificios patrimoniales de la ciudad han sido muy elevados; pero, además, está la propia pérdida emocional — generalmente no valorada— que sufren los habitantes de un área

\footnotetext{
${ }^{2}$ Un informe detallado del terremoto de Lorca puede consultarse en la siguiente web del Instituto Geográfico Nacional http://www.ign.es/ign/resources/sismologia/Lorca.pdf (consulta 30/09/2011).
} 
afectada que ven como su patrimonio ha sido destrozado. La región de Murcia carece de un plan de ordenación del territorio para la reducción del riesgo sísmico; aspecto que debería ser obligatorio en un territorio de elevada peligrosidad sísmica como éste. Debe exigirse la elaboración de cartografía de microzonación sísmica en los municipios de mayor riesgo del sureste ibérico.

6.- Un aspecto que ha quedado de manifiesto, es la rápida respuesta que ha recibido la catástrofe por parte de los organismos y cuerpos encargados de la acción post-evento extremo. España tiene un magnífico sistema de gestión de las emergencias. Los cuerpos y fuerzas de seguridad del Estado, con la Unidad Militar de Emergencias al frente, junto a la policia, bomberos y protección civil local han tenido un comportamiento impecable en los momentos posteriores a los terremotos. Ha habido una coordinación precisa entre ellos y no ha importado que unos dependiesen del Ayuntamiento, de la Comunidad Autónoma o del Estado (vid. figura 3). En la actualidad, España es uno de los países europeos que tiene mejor establecidos los protocolos de actuación postcatástrofe. Es cierto que como territorio de riesgo está obligado a ello, pero debe reconocerse que la labor de protección civil llevada a cabo desde los años ochenta del pasado siglo resulta impecable en la fase del desastre que afecta realmente a los ciudadanos.

7.- Por último, se ha puesto de manifiesto que carecemos de educación para el riesgo en nuestro país. En territorios de riesgo — como Lorca- es im-

FIGURA 3

\section{ACTUACIÓN DE LOS CUERPOS DE SEGURIDAD EN LORCA,} 11 DE MAYO DE 2011

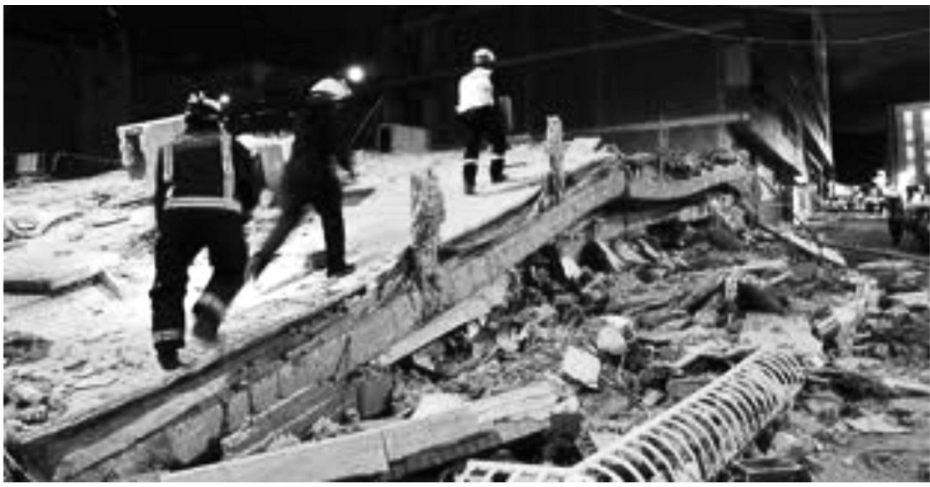

Fuente: www.inforriesgos.es. 
prescindible que la población reciba información, cursos, etc. sobre cómo actuar en caso de catástrofe. Varias víctimas de este terremoto se han producido por salir a la calle nada más notar el primer seísmo, cuando cornisas o mobiliario urbano se desprenden y caen al suelo. Esto es lo último que debe hacerse. Pero la población no lo sabía. En una sociedad de riesgo la información es fundamental para la reducción del riesgo. Una sociedad bien informada, que sepa como reaccionar ante un evento natural de rango extremo, es siempre una sociedad más segura. Esta es una de las piezas que falla en medidas de reducción del riesgo que se han puesto en marcha en nuestro país en las tres últimas décadas. Una vez más, como viene siendo habitual últimamente cuando acontece un evento natural extremo, las redes sociales han funcionado de modo ejemplar, intentando suplir en el momento del desastre la labor de educación para el riesgo que deberían haber llevado a cabo previamente las autoridades.

Cuando acontece una catástrofe natural puede resultar cómodo señalar fallos o errores cometidos, pero lo peor es comprobar que, cuando éstos se indican y resulta razonable su puesta en marcha, las autoridades no hacen caso y hay que esperar al siguiente desastre para proponer soluciones. Se pone así de manifiesto la falta de voluntad del cumplimiento de un principio constitucional básico: la obligación de atender a la seguridad de las personas. El terremoto de Lorca ha vuelto a mostrar que en materia de reducción del riesgo nuestro país ha avanzado en investigación de la peligrosidad y gestión de las emergencias, pero queda por desarrollar lo fundamental: el diseño medidas para reducir la vulnerabilidad y la exposición desde la planificación territorial y la educación para el riesgo a la sociedad que vive en espacios geográficos de elevada peligrosidad natural.

Jorge Olcina Cantos Instituto Interuniversitario de Geografía Universidad de Alicante 